\title{
Difluoroethane Inhalant Abuse, Skeletal Fluorosis, and Withdrawal
}

Adam Custer, MD; Andrew Corse, MD; and Sondra Vazirani, MD, MPH

Prolonged or excessive use of the central nervous system depressant difluoroethane, which is an easily acquired and inexpensive volatile substance that can be inhaled recreationally, is associated with toxicity, and abrupt cessation can induce withdrawal.

\author{
Adam Custer is a \\ Resident Physician in the \\ Department of Medicine, \\ and Andrew Corse is a \\ Resident Physician in the \\ Department of Psychiatry, \\ both at the University of \\ California Los Angeles. \\ Sondra Vazirani is an \\ Attending Physician at the \\ VA Greater Los Angeles \\ Healthcare System. \\ Correspondence: \\ Sondra Vazirani \\ (sondra.vazirani@va.gov)
}

$\mathrm{D}$ ifluoroethane (DFE) is an easily acquired and inexpensive volatile substance that can be inhaled recreationally. ${ }^{1}$ It is found in common household items, including compressed air dusters, refrigerants, and propellants. DFE is a central nervous system (CNS) depressant associated with a brief sensation of euphoria when inhaled. ${ }^{2}$ Prolonged or excessive use is associated with toxicity, and abrupt cessation can induce withdrawal. ${ }^{3-5}$ We present a case of DFE abuse associated with skeletal fluorosis and withdrawal psychosis.

\section{CASE PRESENTATION}

A 39-year-old man with a 6-month history of inhaling 20 to 25 cans of DFE per day presented to the emergency department after abruptly stopping use 6 days prior. He described irritability, agitation, auditory hallucinations, and delusions of "demons trying to harm him."

On presentation, the patient was afebrile with a mild sinus tachycardia. He was calm and cooperative but reported delusions and auditory hallucinations. He denied suicidal or homicidal ideation. His physical examination was remarkable for bony deformities of his hands (Figure 1).

The initial workup included a complete blood count; basic metabolic panel; liver function tests; urine toxicology; and testing for hepatitis $\mathrm{B} / \mathrm{C}$ and $\mathrm{HIV}$; all unremarkable. Psychiatry and poison control were consulted, and he was admitted.

After 72 hours, the patient's irritability, agitation, and sinus tachycardia resolved; however, his psychosis and hallucinations persisted. He was started on olanzapine and transferred to inpatient psychiatry. Additional laboratory tests revealed a serum fluoride of $0.35 \mathrm{mg} / \mathrm{L}$ (normal, 1-47 ug/L), C-telopeptide of $2,663 \mathrm{pg} / \mathrm{mL}$ (normal, $70-780 \mathrm{pg} / \mathrm{mL}$ ), and hand X-rays showing diffuse bilateral periosteal reaction in the phalanges and distal ulnas (Figure 2). ${ }^{6}$

\section{DISCUSSION}

DFE acts as a CNS depressant via glutamate and $\gamma$-aminobutyric acid receptors, causing a brief euphoria when inhaled. ${ }^{2}$ Acute toxicity can cause nausea, vomiting, abdominal pain, and altered mental status. Severe complications include loss of consciousness, mucosal frostbite, angioedema, cardiac arrhythmias, and skeletal fluorosis., ${ }^{2,7}$

Skeletal fluorosis is a rare ramification of excessive or prolonged DFE inhalation. DFE is metabolized into a fluorinated compound that accumulates and leaches calcium from bone, altering its structure. This can manifest as bony deformities with diffuse periosteal reaction and elevated serum fluoride levels. Furthermore, the elevated C-telopeptide level seen in this case may suggest increased bone turnover.

Approximately 50\% of patients report withdrawal symptoms, but the timing, duration, and associated symptoms are not well understood. ${ }^{3}$ Withdrawal can include tremors, diaphoresis, nausea, vomiting, depression, anxiety, irritability, psychosis, and hallucinations. Symptoms typically start within 24 to 48 hours of cessation and last for 3 to 7 days. ${ }^{5}$ Psychotic symptoms often abate quickly; however, anxiety and insomnia can persist for weeks. ${ }^{5}$ There are no formal treatment guidelines, but poison 
FIGURE 1 Bony Deformities

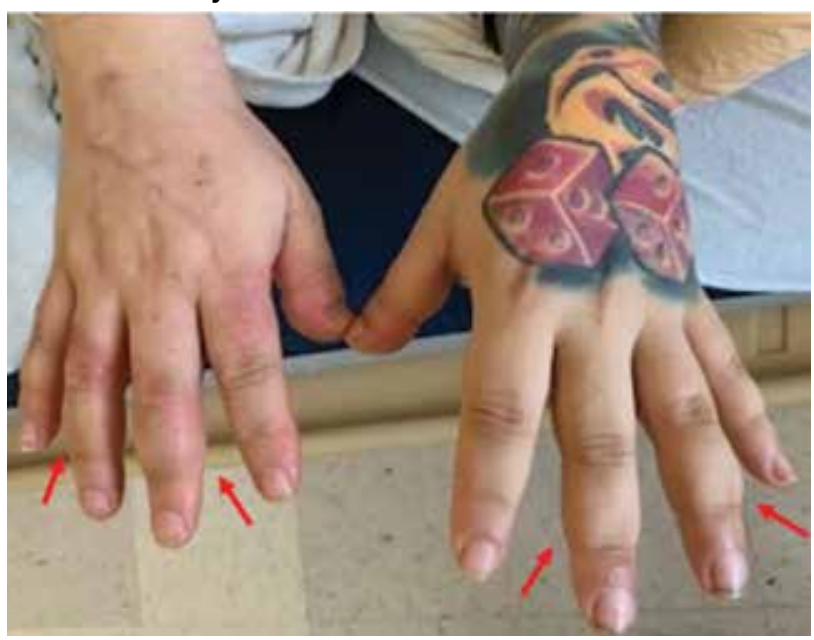

Bilateral hand deformities of the phalanges are indicated by the red arrows.
FIGURE 2 Hand X-ray

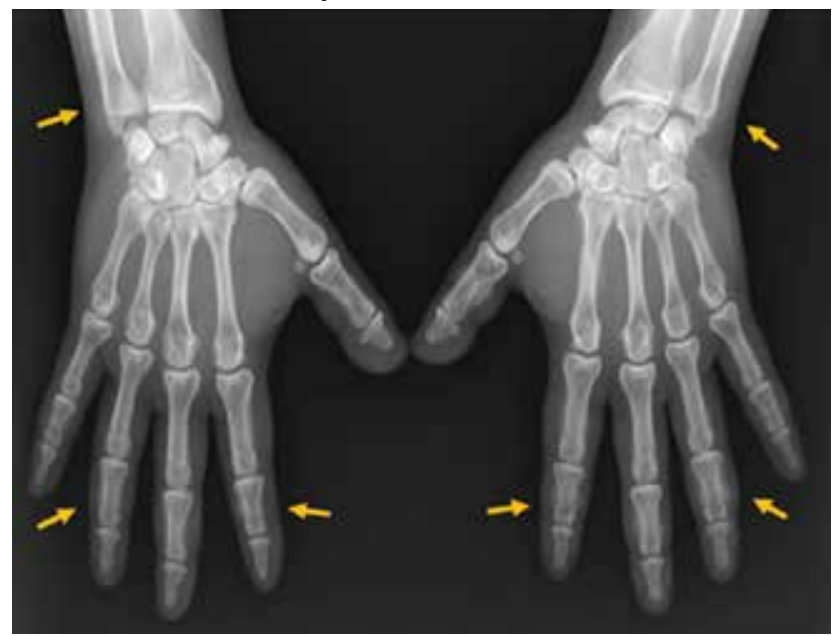

Hands X-ray orange arrows show diffuse periosteal reaction of the phalanges and distal ulnas consistent with skeletal fluorosis. control suggests observation and as-needed benzodiazepines. Although this patient's irritability and agitation resolved, his psychosis and hallucinations persisted, raising concern for an underlying psychiatric diagnosis and prompting transfer to inpatient psychiatry.

\section{CONCLUSIONS}

Health care providers should recognize the symptoms of DFE toxicity, its complications, and withdrawal. Collaborating with psychiatry and poison control is beneficial in providing guidelines for supportive care.

\section{Author disclosures}

The authors report no actual or potential conflicts of interest with regard to this article.

\section{Disclaimer}

The opinions expressed herein are those of the authors and do not necessarily reflect those of Federal Practitioner, Frontline Medical Communications Inc., the US Government, or any of its agencies. This article may discuss unlabeled or investigational use of certain drugs. Please review the complete prescribing information for specific drugs or drug combinations-including indications, contraindications, warnings, and adverse effectsbefore administering pharmacologic therapy to patients.

\section{References}

1. Arroyo JP, Johnson DC, Lewis JB, et al. Treatment of acute intoxication from inhaled 1,2-difluoroethane. Ann Intern Med. 2018;169(11):820-822. doi:10.7326/L18-0186

2. National Library of Medicine, PubChem. Hazardous Substance Data Bank (HSDB) 1,1-Difluoroethane. https:// pubchem.ncbi.nlm.nih.gov/source/hsdb/5205. Updated October 25, 2016. Accessed May 20, 2020.

3. Perron BE, Glass JE, Ahmedani BK, Vaughn MG, Roberts DE, Wu LT. The prevalence and clinical significance of inhalant withdrawal symptoms among a national sample. Subst Abuse Rehabil. 2011;2011(2):69-76.

doi:10.2147/SAR.S14937

4. Perron BE, Howard MO, Vaughn MG, Jarman CN. Inhalant withdrawal as a clinically significant feature of inhalant dependence disorder. Med Hypotheses. 2009;73(6):935-937. doi:10.1016/j.mehy.2009.06.036

5. Addiction Center. Inhalant withdrawal and detox. https://www.addictioncenter.com/drugs/inhalants /withdrawal-detox. Accessed May 18, 2020.

6. Torra M, Rodamilans M, Corbella J. Serum and urine ionic fluoride: normal range in a nonexposed population. Biol Trace Elem Res. 1998;63(1):67-71. doi:10.1007/BF02785278

7. Cohen E, Hsu RY, Evangelista P, Aaron R, Rubin LE. Rapid-onset diffuse skeletal fluorosis from inhalant abuse: a case report. JBJS Case Connect. 2014;4(4):e108. doi:10.2106/JBJS.CC.N.00085 